\title{
Estudo comparativo de pesquisas quantitativas e qualitativas sobre a influência da motivação na escolha de curso universitário.
}

\author{
Estudio comparativo de investigaciones cuantitativas y cualitativas
} sobre la influencia de la motivación al escoger una profesión universitaria Comparative study of quantitative and qualitative research on the
influence of motivation when choosing a university profession

Yulimer Uzcátegui ${ }^{1}$ Gionara Tauchen ${ }^{2}$

\begin{abstract}
Resumo
Considerando a complexidade que envolve a escolha de curso universitário, o seguinte estudo tem como objetivo comparar as pesquisas quantitativas e quantitativas sobre motivação na escolha de cursos universitários a partir de um estudo de natureza bibliográfica. Encontrando majoritariamente estudos quantitativos, desenvolvidos por meio de escalas apoiadas em fundamentações teóricas como: dicotomia entre intrínseco e extrínseco, o modelo continuum de autodeterminação, teoria expectação/valor o teoria de Metas. Por sua parte, as pesquisas com uma abordagem qualitativa sobre motivação são poucas, no entanto, deixam observar como emergem as atividades associadas aos tipos de motivação que se discutem em diferentes fundamentos teóricos. A comparação entre os enfoques metodológicos permitiu observar que os estudos quantitativos utilizavam escalas tipo likert para converter as variáveis, fundamentadas em teorias especificas, em dados quantificáveis que geram resultados estadísticos, os quais podem ser comparados com a teoria para fazer explicações na realidade observada. Os estudos qualitativos, recoletam informação utilizando entrevistas semi-estruturadas, as quais foram analisadas pelo analises de conteúdo, podendo compreender a realidade a partir das categorias emergentes e os fundamentos teóricos que apoiam e contribuem com as explicações e validação das categorias
\end{abstract}

Palavras-Chave: motivação, enfoques quantitativos, enfoques qualitativos

\section{Resumen}

Considerando la complejidad que envuelve escoger una carrera universitaria, el presente estudio tiene como objetivo comparar las investigaciones cuantitativas y cualitativas sobre motivación en la selección de carreras universitarias utilizando mediante un estudio de naturaleza bibliográfica. Encontrando mayormente estudios cuantitativo, basados en escalas apoyadas en fundamentos teóricos como: dicotomía entre intrínseca y extrínseca, el modelo continuum de autodeterminación, teoría expectación/valor o teoría de Metas. Por otra parte, las investigaciones con un abordaje cualitativo sobre motivación son pocas, sin embargo dejan observar la emergencia, de actitudes asociadas a los tipos de motivación que se discuten en diferentes fundamentos teóricos. La comparación entre los enfoques metodológicos permitió observar que los estudios cuantitativos utilizaban escalas tipo likert para convertir las variables, fundamentadas en teorías especificas, en datos cuantificables que generan resultados estadísticos, los cuales pueden ser contrastados con la teoría para poder lograr explicaciones a la realidad observada. Los estudios cualitativos, recolectaron información mediante entrevistas semiestructuradas, las cuales fueron analizadas por un análisis de contenido, logrando comprender la realidad a partir

\footnotetext{
${ }^{1}$ Mestrando Programa de Pós-Graduação em Educação em Ciências: Química da Vida e Saúde. Universidade Federal do Rio Grande (FURG). Rio Grande do Sul. Brasil. E-mail: yvu789@ gmail.com

${ }^{2}$ Doutora em educação pela Universidade Federal do Rio Grande (FURG). Rio Grande do Sul. Brasil. E-mail: giotauchen@gmail.com
} 
de categorías emergentes y los fundamentos teóricos que sustentan y contribuyen con las explicaciones y validación de las categorías.

Palabras claves: motivación, enfoque cuantitativo, enfoque cualitativo

\begin{abstract}
Considering the complexity involved in choosing a university career, this study aims to compare quantitative and qualitative research on motivation in the selection of university careers using a study of a bibliographic nature. Finding mainly quantitative studies, based on scales supported by theoretical foundations such as: dichotomy between intrinsic and extrinsic, the continuum model of self-determination, expectation / value theory or Goals theory. On the other hand, the researches with a qualitative approach on motivation are few, nevertheless they let observe the emergency, of attitudes associated to the types of motivation that are discussed in different theoretical foundations. The comparison between methodological approaches allowed observing that quantitative studies used likert scales to convert variables, based on specific theories, into quantifiable data that generate statistical results, which can be contrasted with the theory in order to achieve explanations of the observed reality. On the other hand, qualitative studies collected information through semi-structured interviews, which were analyzed by a content analysis, managing to understand reality from emerging categories and the theoretical foundations that support and contribute with the explanations and validation of the categories.

Keywords: motivation, quantitative approach, qualitative approach
\end{abstract}

\title{
1. Introducción
}

Seleccionar una profesión universitaria es una decisión importante que marca el futuro en la vida de cualquier persona. Muchas veces ocurre en el tiempo de pubertad, donde se presentan, según Bravo y Vergara (2018), constantes cambios psicológicos, como inseguridades, deseos de libertad y cambios de intereses. Así mismo, está asociada con surgir socialmente y llegar a ser alguien en la vida, en otras palabras, involucra la proyección de metas, sueños y visión de lo que se desea ser. Aunado a esto Figueroa (1993 p. 5), nos dice lo siguiente:

El ingreso a la Universidad coincide con la orientación del "Yo" hacia el porvenir, la relación con el núcleo familiar ha variado sustantivamente y el colegio se considera "el pasado". Cada joven que ingresa a la Universidad se transforma en una promesa individual e invalorable, que se cumplirá si se forma adecuadamente, no sólo desde el punto vista académico profesional, sino desde el punto de vista de los valores y del servicio a la comunidad. El ingreso a la Universidad coincide con el carácter cada vez más abstracto de la inteligencia y con la voluntad más consciente y precisa de conseguir logros.

Por otra parte, Kilinç, Watt y Richardson (2012), apoyados en psicólogos vocacionales consideran que los factores motivacionales por los cuales se decide una carrera profesional, no siempre son totalmente racionales. Las habilidades, intereses, valores, opciones, consejos y opiniones de personas cercanas pueden influenciar la decisión, así como la comunidad a la que pertenece y los impactos en el mercado laboral. 
Entre los aspectos que están envueltos en esta decisión, según Cano (2008) están sus intereses, aptitudes, información profesiográfica (profesiones de interés para la zona donde se desenvuelve) y especialmente resalta la importancia de la motivación, postulando que la falta de motivación para los estudios genera apatía para el acercamiento hacia las implicaciones de la selección de una carrera profesional (personales, laborales y sociales), consecuentemente presentara ausencias motivacionales para desenvolver sus estudios.

Por otra parte, Bzuneck y Guimarães (2007), indican que los estudiantes motivados (intrínsecamente), alcanzan su autoformación, valorizan las actividades por sí mismas, incluyen un componente afectivo (interés), generando comprometimiento en la ejecución de tareas, persistencia e capacidad de concentración (desligado al tiempo). Considerando autoformación como el impulso personal para llevar a cabo tareas, la iniciativa propia por aprender, vinculada con la formación permanente y con la búsqueda de recursos en su entorno, para satisfacer los intereses personales de su formación. (SARRAMONA, 1999).

En este sentido, resulta importante conceptualizar la palabra formación, la cual, apoyándose en Pineau (2003, p. 156), y tomando en cuenta que para él la formación "se inscribe en un movimiento transdisciplinar de tentativas de tratamientos de la multicausalidad", se podría definir como un proceso continuo de transformación del sujeto por acción de su desenvolvimiento interpersonal y su interacción con su entorno social.

Así mismo, considerando el sujeto envuelto en la complejidad del desenvolvimiento de toda su vida, se presentan aspectos hetero y homogeneizadores de forma simultánea. Dando origen a una acción autoformadora y autotransformadora emergente de fuerzas capaces de condicionarse y moldearse entre fuerzas semejantes (PINEAU, 2003).

Existen tres movimientos de formación, los cuales representan esas polaridades complejas que emergen de esas fuerzas de acciones. Teniendo la autoformación, como polo individual, el cual provoca un movimiento subjetivo de formación y depende de las fuerzas del protagonismo que se ejerce en la formación. La heteroformación, corresponde al polo social y está ligado a las acciones sociales intencionales, de un aprendizaje en conjunto, a partir del proceso educativo mutuo, está siempre en movimiento con la autoformación. El tercer movimiento corresponde a la ecoformación, es un polo discreto y silencioso; corresponde a la base que permite tanto la autoformación, como a heteroformación; este movimiento destaca la reciprocidad de formación en los diversos ambiente donde el sujeto se desenvuelve, no solo institucional. (PINEAU, 2003).

Este proceso complejo permiten adquirir conocimiento, a la vez que se reorganiza el propio principio del saber, en medio de permanentes cambios que transforman la formación, 
inmersos en un “(...) proceso permanente, dialectico y multiforme de colocación en conjunto y sentido (...)”.(PINEAU, 2003, p. 164). En el entorno de formación de los estudiantes, Steimann, Bosch y Arassa (2013), indican que debe existir en los estudiantes la voluntad de aprender y que esta se relaciona con sus propias experiencias, las cuales darán valor y relevancia a la acción de estudiar o hacer actividades académicas, modificando la disposición en su acción. Considerando que, según Pintrich (2003), la motivación responde, en parte, a situaciones externas, condiciones físicas, percepciones o representaciones mentales de la situación, además de la influencia de su entorno familiar, social y cultural, entre otros factores que pueden modificar la dinámica escolar.

Entre las teorías que ayudan a conocer e interpretar la motivación tenemos la Autodeterminación, la Autorregulación, Modelo de metas y el modelo de expectación/valor. Cada una de ellas ha contribuido en diferentes estudios sobre la motivación escolar y en la selección de profesión universitaria.

Por una parte, la autodeterminación, según García, Garduño y Organista (2006), indican que en motivación asociada a la elección de carrera, están involucrados aspectos tanto intrínsecos como extrínsecos. En cuanto a la motivación intrínseca tenemos la autorrealización (satisfacción propia) y motivos altruistas. La autorrealización, esta se refiere a la participación en actividades que son de su agrado e interés por sentir afinidad a ellas; probablemente estén ligadas a una profesión, generando interés en su estudio. Por otro lado, se tienen los motivos altruistas, que se refieren a las personas que están a disposición de ayudar a otros, procuran trabajos al servicio de los demás, en la búsqueda de un bienestar colectivo, están comúnmente asociado a carreras de la salud y educadores.

Bzuneck y Guimarães (2007), sugieren que las formas de motivación autodeterminadas, así como la propia motivación intrínseca, dependen positivamente del comportamiento interpersonal caracterizadas como promotores de autonomía, ya que genera un desarrollo completo en las tareas, persistencia, concentración, desconexión con el tiempo invertido en la realización y placer.

La motivación extrínseca, según Montalvo-Aguilar et al (2013), presenta aspectos relacionados a antiguos docentes, movilidad social, la influencia familiar (como presión de los padres), recomendaciones, incentivos externos (asignaciones, posibilidades de estudio, becas) y económicos. Estos factores se reflejan en no tener claro lo que se desea estudiar, selecciona algo en lo que no quiere trabajar, cambios de carrera, deserción universitaria, deseos por satisfacer necesidades principalmente económicas. 
Sin embargo, existen factores externos que condicionan el ingreso universitario y son las políticas públicas de educación superior de cada país y de los criterios internos de cada universidad, las que crean modalidades de ingresos que pueden o no beneficiar la selección de la población estudiantil. Por ejemplo, en Argentina, a partir de 1983 la modalidad de acceso más extendida en las universidades públicas es el ingreso directo, siendo el diploma de culminación de estudios de secundaria el único requisito. Sin embargo, academias de las universidades públicas han adaptado modalidades para realizar una elección de sus futuros estudiantes, como pruebas internas. En las universidades privadas se realizan formalmente examen de ingreso que sirven de selección o de diagnóstico.(CHIROLEU, 2009).

En Venezuela, se han dado diferentes formas de ingreso universitario. Desde el año 1984 hasta el 2007, el Estado venezolano, a través de la Oficina de Planificación del Sector Universitario (OPSU), se implementó el Sistema Nacional de Admisión para el ingreso al Subsistema de Educación Superior, en el cual se centralizaban todas las preinscripciones y que constaba de una Prueba de Aptitud Académica (con un apartado de razonamiento verbal y otro en habilidad numérica) y la posibilidad de seleccionar tres opciones de carrera, además consideraba datos socioeconómicos. Cada estudiante obtiene un Índice Académico de Ingreso, el cual resulta de la suma porcentual de calificaciones del Bachillerato (Educación Media), hasta cuarto año para los cursantes del último, con un $60 \%$ y el puntaje obtenido en la Prueba de Aptitud Académica en un 40\%. Este índice permite clasificar a los estudiantes y distribuirlos entre instituciones y carreras según los cupos que las mismas establezcan.(PÉREZ y DÍAZ, 2006).

Este sistema de ingreso fue abolido en el año 2007. Según Elvira-Valdés y Pujol (2014), ese sistema de acceso a la universidad era excluyente y discriminatorio. Posteriormente, en el 2009, se prohibieron las pruebas voluntarias de ingreso en las universidades nacionales. Actualmente la preinscripción se realiza por el portal de la OPSU (http://ingreso.opsu.gob.ve/) teniendo como único requisito las notas de bachillerato (igualmente, hasta cuarto año para los cursantes del último año), datos socioeconómicos y tiene seis opciones de selección de carrera universitaria o Programa Nacional de Formación (PNI).

Por otra parte, en Brasil se realiza el "Examen Nacional de Ensino Medio" (Enem), que es organizado por el Instituto Nacional De Estudos E Pesquisas Educacionais Anísio Teixeira (INEP). En dicho evaluación se presenta una prueba sobre redacción de lengua portuguesa y 4 pruebas objetivas (cada una con 45 ítems de selección múltiple), las cuales están asociadas a las siguientes áreas del conocimiento: lenguaje, códigos y su tecnología, educación física y tecnologías de información y comunicación, ciencias humanas y sus tecnologías, ciencias 
naturales y sus tecnologías, matemática y sus tecnologías. (DIARIO OFICIAL DA UNIÃO, 2018).

Una vez presentada la prueba comienzan los procesos selectivos mediante el Sistema de Selección Unificada (SiSU), el cual es un sistema informático del Ministerio de Educación que ofrece cupos en las instituciones públicas de educación superior a los participantes del Enem. Cada estudiante puede optar a dos opciones y las puede cambiar durante el proceso de inscripción, el proceso selectivo ocurre en función de la calificación que alcanzo durante el proceso de pruebas. (SiSU, 2018) Este sistema de acceso, por SiSU depende de la participación de las universidades en el sistema. Generalmente las instituciones públicas se suman al SiSU, sin embargo, algunas continúan aplicando sus propios exámenes de selección y, muchas instituciones privadas utilizan una prueba de redacción como forma de ingreso.

En cuanto al ingreso universitario facilitado, Griner et al. (2015) indica que existen grupos en oposición, indicando que genera una disminución en la calidad educativa de las universidades federales. Por otro lado, existen sectores sociales que apuntan a la aprobación de un análisis curricular más amplio, validando en desempeño de los alumnos con las notas obtenidas a lo largo de la Educación Media, por ser un tipo de predicción confiable sobre el éxito académico, más que una prueba, siendo así correlacionado con la idea de diversidad e inclusión social.

En Chile, según Garcés y Fuentes (2017), el modo de universalización de la inscripción por medio de la gratuidad, ocurre conforme a la reforma al Sistema Único de Admisión Universitaria (SUA), la cual desde el año 2013 considera el ranking de notas otorgando una mayor ponderación al desempeño educativo, revalorizando la trayectoria escolar y el esfuerzo en la Educación Media por parte del alumno. Además de una Prueba de Selección Universitaria (PSU), que tiene como objetivo prever la adaptación universitaria en función de sus capacidades o conocimiento adquiridos en su Educación Media.

El PSU muestra en su site http://www.psu.demre.cl todo el proceso de inscripción, en el se denota que consta de 4 pruebas, de las cuales, dos son obligatorias y corresponden a Matemática y a Lenguaje y Comunicación. Las otras dos son electivas y corresponden a Ciencias (Biología, Física, Química y Técnico Profesional) e Historia, Geografía y Ciencias Sociales. Posteriormente con el puntaje final entre las notas y los resultados de las pruebas y considerando los puntajes de ponderación para las diferentes carreras universitarias que ofrecen las universidades del país, se da la postulación a las carreras que sean de su agrado, en función de las opciones que tenga por el puntaje obtenido. 
Al observar los diferentes procesos selectivos que realizan los estudiantes que desean optar a un cupo universitario, vale la pena conocer lo que los motivó a persistir y alcanzar esta meta en determinada profesión, especialmente sobre la motivación a seguir estudiando y la influencia que ha tenido su periodo escolar en esta decisión. En función de esto, se propone el siguiente objetivo de investigación: comparar el enfoque cualitativo y cuantitativo usado en investigaciones sobre la influencia motivacional en la elección de una profesión universitaria basados en un estudio de naturaleza bibliográfica.

\section{Metodología}

El enfoque investigativo organiza la investigación, por lo tanto es mucho más que técnicas y análisis de recolección de datos. Su naturaleza dependerá de los problemas que atañen al investigador o del fenómeno a estudiar en sí. En este caso, al momento de estudiar la motivación, se considera una lista de comportamiento que debe tener una persona, ante una actividad específica, para poder pertenecer a una clasificación en los tipos de motivación, basados en un fundamento teórico determinado. Como resultado de esta visión, se han desarrollado diversos estudios sobre motivación bajo un enfoque cuantitativo.

Los estudios basados en un enfoque cuantitativo, sean de orden inductivo o deductivo, tienen como punto de partida la teoría, que enmarca las posibilidades de formular explicaciones acerca de algún aspecto de la realidad. Exige una recolección de datos sistemática, capacidad de percibir la importancia de los datos colectados, actualización sistemática e adición de nuevas ideas y teorías. Tomando en consideración que la descripción matemática es su lenguaje y la estadística su soporte para establecer la o relación entre el modelo teórico propuesto y los datos observados de la realidad, permitiendo la descripción de las causas de un fenómeno, la relación entre las variables, entre otros aspectos. (TEIXEIRA, 2014).

También se han elaborado estudios sobre motivación bajo un enfoque cualitativo, la cantidad es muy poca, sin embargo muestran otro abordaje para percibir el fenómeno a estudiar. Esto se debe a que el enfoque cualitativo enfatiza en el proceso como ocurre un fenómeno, busca comprender el contexto de la situación problemática, es decir, se fundamenta en un análisis fenomenológico y/o interpretativo, donde el interés se da en la descripción e interpretación de una realidad. Dicha realidad se estudia reduciendo la distancia entre la teoría y los datos encontrados del fenómeno en estudio; dichos datos se obtienen a partir de diferentes técnicas o fuentes, pero siempre desde la óptica de las personas que 
interactúan con la realidad en estudio. Esto hace que se favorezca la peculiaridad y le da un carácter rico, holístico e real del fenómeno en cuestión. (TEIXEIRA, 2014).

\section{Enfoques metodológicos e instrumentos para conocer sobre motivación}

Son varios los estudios que se han preocupado por la motivación a nivel universitario. Algunos en función de continuar la carrera, otros en función de la participación en clase, algunos sobre la motivación referente a una profesión específica. Cada uno presenta escalas que determinan cuantitativamente lo que motiva o no a los estudiantes participantes. Estas escalas son el fundamento para la realización de cuestionarios, los cuales, como toda técnica cuantitativa, traducen el fenómeno social en números, teniendo que la calidad de los datos que se analizan le da calidad a la investigación y que esto depende de la adecuación de cada una de las preguntas formuladas en el cuestionario con los objetivos del estudio. (CANALES, 2006).

Así mismo, para la realización del cuestionario es necesario el proceso de operacionalización de las variables presentes en el estudio, logrando definir cuidadosamente los conceptos que se pretenden medir y los fenómenos que intervienen en los conceptos y son observables, logrando tener indicadores medibles, que se transformaran en preguntas o ítems para poder obtener datos. (CANALES, 2006).

A modo de resumen sobre algunos fundamentos teóricos aplicados en encuestas, se presenta la figura 1 para posteriormente detallar cada propuesta.
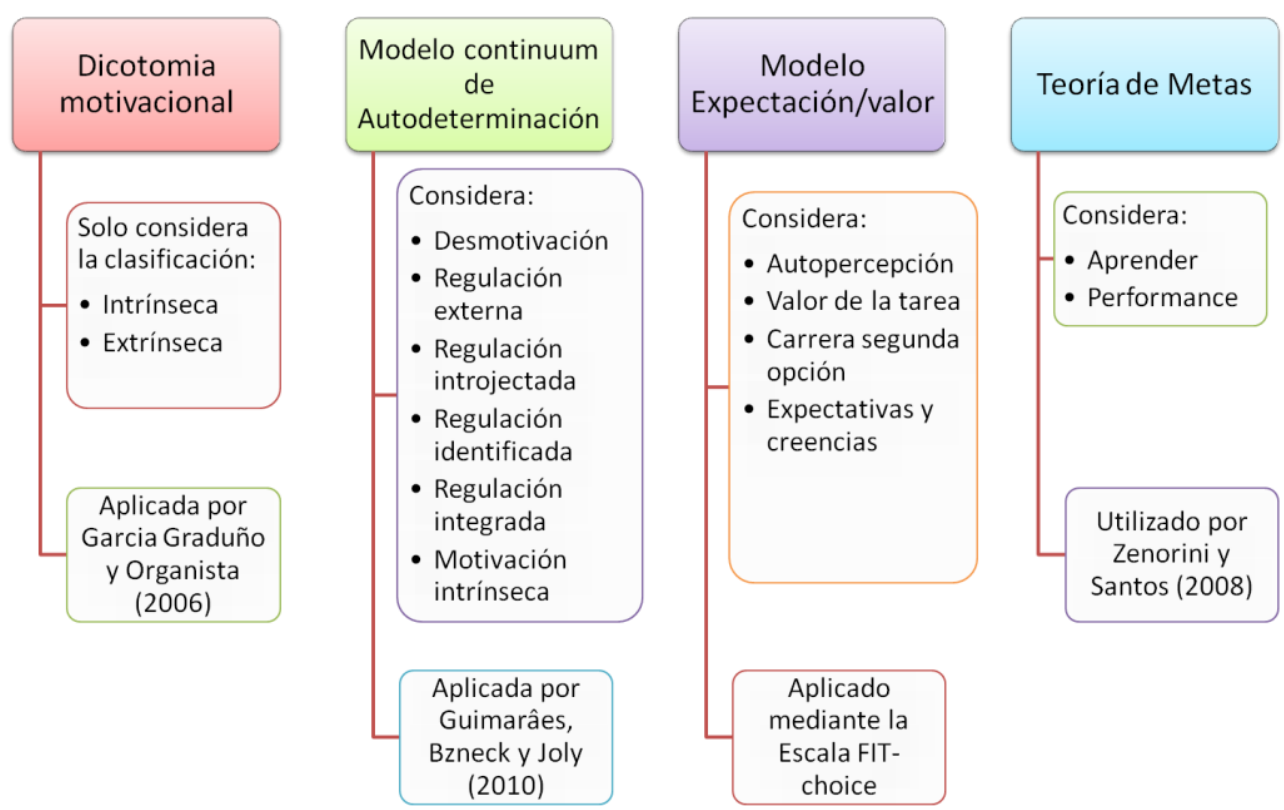

Figura 1. Encuestas utilizadas por investigadores en función de teorías motivacional. 
Utilizando la dicotomía entre intrínseco y extrínseco, García Garduño y Organista (2006), han realizado un estudio sobre los motivos que llevaron a los estudiantes a formarse para ser maestro de educación primaria y denominada como "normal”. Las preguntas estaban direccionadas en conocer si se tienen motivos personales o por influencia externa para la selección, si realmente desea estudiar la carrera y terminarla, si desearía estudiar otra profesión, si desea ejercer la profesión y si siente que es su vocación.

Por otra parte, Joly y Prates (2011), se basaron en el modelo continuum de autodeterminação de Reeve, Deci y Ryan (2004) en el cual se presentan 6 etapas, las cuales se organizan de la siguiente manera: desmotivación (ausencia de ganas para realizar una actividad específica), regulación externa (presiones externas, controlada por restricciones o recompensas), regulación introyectada (internalización de valores, aun con control externo), regulación identificada (realización de actividades y reconocimiento de su importancia para acciones futuras), regulación integrada (refleja un alto control interno y es congruente entre el yo y los propios valores y objetivos), motivación intrínseca (deseo de hacer algo para sentir satisfacción).

En su estudio utiliza la Escala de Motivación Académica diseñada por Guimarães, Bzuneck \& Joly (2010, p.178), la cual tiene por objetivo caracterizar la autopercepción de los estudiantes universitarios en referencia a su motivación para frecuentar la universidad.

Ambos estudios postulan escalas enmarcadas en conocer la motivación relacionada al estudio de una profesión universitaria, considerando los tipos de motivación intrínseca y extrínseca que pueden ser determinadas mediante preguntas con respuestas tipo Likert, donde se evidencie el juicio de valores de las personas que participaron en dichas encuestas.

En cuanto al modelo expectación/valor ampliamente explicado por Eccles y Wigfield (1992), surge la escala FIT-choice (Factors Influencing Teaching choice), creado por Watt y Richardson (2007), la cual ha sido traducida a varios idiomas y adaptada a diferentes contextos sociales, sus alcances han llevado a tener su propio site, http://www.fitchoice.org/, en el cual se observan diferentes investigaciones aplicando y adaptando dicha escala. Ha sido traducida, según Suryani et al. (2016), al turco (aplicación en Turquia en el 2012), mandarin (aplicación en China en el 2012), español (aplicación en España en el 2014), croata (aplicación en Croacia en el 2012), alemán (aplicación en Alemania en el 2012), al francés (aplicación en Suiza en el 2012) y en el 2016 traducido al indonesio, durante la investigación de Suryani et al. (2016)

En el FIT-choice se describe cuatro (4) elementos que van a influir en la selección de carrera, esos son: 
a. Auto percepción: habilidad para explicar lo que se sabe.

b. Valor de la tarea, que está orientado a una utilidad personal (seguridad, movilidad laboral, conciliación familiar), o a una utilidad social (contribución social, mejoras en la sociedad).

c. Carrera de segunda opción, correspondiente a una selección por descarte.

d. Expectativas y creencias, relacionado con la exigencia de la carrera y de la profesión al momento de ejercer.

El marco FIT-Choice busca conocer las experiencias previas de enseñanza y aprendizaje, influencias sociales y sociales disuasión involucradas en la elección de una carrera docente. En la influencia social están envueltos los familiares y amigos; la disuasión social se refiere a las influencias de otros para no elegir una carrera.

Su conexión con la teoría expectación-valor, según Suryani, Watt y Richardson (2016 p.182), viene dada por las autopercepciones sobre las habilidades de enseñanza percibidas; las tareas (tanto las demandas como su retorno). La demanda de tareas permite conocer la visión con respecto a los maestros (considernado nivel de experiencia y carga de trabajo). El retorno de la tarea, se observa por el valor dado a enseñar, es decir, si considera como una profesión respetada. Los aspectos relacionados a valor de la tarea, valor de utilidad social y valor de utilidad personal, Suryani, Watt y Richardson (2016 p.182), nos los explica de la siguiente manera:

Medidas de valor intrínseco los intereses personales de los participantes y el placer de trabajar como maestro. Valor de utilidad social evalúa el deseo de los futuros docentes de contribuir positivamente a la sociedad trabajando como docentes (que contiene factores de primer orden: forma el futuro de los niños / adolescentes, mejora social equidad, hacer contribuciones sociales y trabajar con niños / adolescentes). Utilidad personal el valor consiste en la seguridad laboral, el tiempo para la familia y la transferibilidad laboral

Los factores tomados en cuenta en la escala FIT-choice, son presentados por Kilinç, Watt y Richardson (2012 p.222-225), estos son: habilidades para enseñar, valor intrínseco de la carrera, segunda opción de carrera (la educación no era la primera opción de carrera), seguridad laboral, tiempo para la familia, movilidad laboral; visión de: formar a niños (as)/adolescentes, mejorar la equidad social, hacer contribución social; trabajar con niños/adolescentes, influencia social, antiguos profesores y experiencias de aprendizaje, alta demanda, estatus social, experiencia en la carrera, salario, disuasión social y satisfacción con la elección. 
Otros de los modelos empleados en la determinación de factores motivacionales en la selección de una profesión es el de Metas. Este modelo es definido por Ames (apud SANTOS, ALCARA Y ZENORINI, 2013 p. 532) como un conjunto de pensamientos, creencias, propósitos y emociones que convierten las expectativas de los estudiantes en relación a la elaboración de tareas. La teoría de metas describe dos tipos de metas, las cuales son aprender y performance.

Teniendo como meta aprender, aquellos estudiantes que buscan su crecimiento intelectual, valoran el esfuerzo personal, son persistentes en la realización de actividades y desafíos, utilizan estrategias de aprendizaje más efectivas, son creativos en la búsqueda de comprensión de los contenidos; para ellos los fracasos o errores son estímulos para buscar nuevas estrategias para lograr los objetivos. Por otro lado, los estudiantes con metas performance se preocupan por demostrar sus capacidades a otros, evitan desafíos o actividades de las que no se sienten seguros; los fracasos son tomados de forma negativa, lo asocian a falta de capacidades, sienten vergüenza y rabia.

En el 2008, Zenorini e Santos desarrollaron una escala de motivación para el aprendizaje Universitario. En el cual se toma en cuenta una tercera dimensión, la meta performanceevitación, relacionada, según Santos, Alcara y Zenorini (2013), a estudiantes que evitan participar por miedo a los otros y a su juicio en función de lo que puede decir o aportar.

Dicha escala cuenta con veintiocho (28) itens, con opciones de respuesta en una escala tipo Likert (de acuerdo, no sé y en desacuerdo). Los itens están estructurados en doce (12) preguntas dirigidas a meta aprender, siete (7) a performance y nueve (9) a performanceevitación. (SANTOS, ALCARA Y ZENORINI, 2013)

Este tipo de estudio teóricamente es completo y está fuertemente sustentado, lo que ha permitido su amplia repetición en diferentes comunidades, homogenizando las realidades, sin considerar el carácter individual, los aspectos sociales y culturales que envuelven cada una de esas comunidades. La observación en común, con los trabajos basados tanto en autodeterminación como en expectación-valor o teoría de metas, es que se fundamentan en una escala tipo likert, donde esbozan toda la teoría y son los porcentajes obtenidos los que explicaran la motivación presente en una población estudiantil. Dejando de lado que la motivación genera acción, se asocia con afectos, con cultura, con interacción social, tal como lo expresa Pintrich (2003).

Para estudiar motivación, es necesario considerar que los individuos tienen un desarrollo multidimensional, tal como lo explica Morin (1999, p.74), donde "la individualidad, la cerebralización, la afectividad, las posibilidades de elección y de decisión, la curiosidad, (...) 
que desarrollan al mismo tiempo el conocimiento y las posibilidades de emancipación del conocimiento, se desarrollan solidaria e interactivamente", la multiplicación de las comunicaciones entre individuos, teje una red social cada vez más compleja, la cual favorece el desarrollo de los individuos y así la complejidad social.

En función de esto, a continuación se presentan las investigaciones sobre motivación, de forma general, debido a la poca cantidad de trabajos fundamentados en un enfoque cualitativo. El cual, según Teixeira (2014), tiene como premisa que lo social es un mundo de significados que sustentan la investigación, y es mediante el lenguaje de los actores sociales y sus interacciones que se obtiene la materia prima para poder analizar la realidad en estudio.

El trabajo de Dos Santos, Rodenbusch y Antunes (2009), buscaba verificar los aspectos motivacionales presentes en los docentes de educación superior, el trabajo está basado teóricamente en la concepción de Dos Santos y Antunes (2007) apud Dos Santos, Rodenbusch y Antunes (2009, p. 295-297), en la cual las personas son intrínsecamente motivadas y sufre modificaciones por motivos extrínsecos, donde cada sujeto es constituido por sus experiencias sociales y culturales particulares. El estudio fue realizado bajo un paradigma naturalista mediante un estudio de casos que conto con una entrevista para siete profesores universitarios de dedicación exclusiva en el área de la salud de la Universidade Privada no estado do Rio Grande do Sul (PUCRS).

Por medio de un análisis de contenido lograron las siguientes categorías: Entendimientos sobre motivación, trayectoria académica: en búsqueda de ser un profesor universitario, historia de vida y autorrealización, formación persona y de futuras generaciones. Consiguieron capturar las concepciones que los docentes tenían sobre motivación y el interés que presentan en contribuir con sus estudiantes para la formación de ciudadanos además de impartir conocimientos, además de sus entendimientos sobre la importancia social que presenta la educación universitaria.

Echeverri-Sánchez et al. (2018) realizaron un estudio cualitativo a partir de 29 entrevista semi estructurada aplicada a estudiantes de la Instituto Tecnológico Metropolitano (ITM), ubicado en la ciudad de Medellín. El diseño metodológico utilizado es de tipo cualitativo a través. Entre los objetivos de las entrevistas se encuentra la motivación para la creación de empresas. El análisis fue realizado toman do en cuenta el análisis de contenido de Graneheim y Lundman (2004) apud Echeverri-Sánchez et al. (2018, p. 8). A pesar de no tener una teoría motivacional de referencia, es observable una consideración dicotómica de motivación intrínseca y extrínseca, ya que mediante el análisis realizado a las entrevistas ellos enfatizan que se observaron dos tipos de motivación. La primera es llamada de propia, en ella 
convergen las metas y los deseos, teniendo como horizonte la independencia y autonómica en asuntos de emprendimiento empresarial. El segundo tipo de motivación es llamado de externo y se refiere a carencias económicas, oportunidades y asuntos laborales, como injusticia y generar empleos.

Mediante la investigación cualitativa descriptiva de Bernal-Becerril y GodínezRodríguez (2016), referente al aprendizaje del cuidado obstétrico de los estudiantes de la Universidad Nacional Autónoma de México, pudieron emerger 2 categorías, entre ellas la motivación. La categoría motivación trajo consigo las características de la motivación escolar, con unidades de significados referentes a autonomía, dedicación, inversión de tiempo y seguridad en las acciones por medio de las prácticas y la comprensión del conocimiento que se debe aplicar. Para dar respaldo a la categoría se baso en la concepción de la motivación para aprender de García (2008) apud Bernal-Becerril y Godínez-Rodríguez (2016, p.237). La recolección de datos se baso en una entrevista semi-estructurada para estudiantes de séptimo semestre de la Licenciatura en Enfermería y Obstetricia y fue analizada mediante un análisis de contenido basados en De Souza-Minayo apud Bernal-Becerril y Godínez-Rodríguez (2016, p. 236)

Davoglio, Spagnolo y Santos (2017), nos traen un trabajo donde se realizaron entrevistas y se analizaron bajo el enfoque de contenido de Bardin (1977), con el apoyo teórico de la autodeterminación de Ryan y Deci (2000). El análisis permitió que emergieran las siguientes categorías: relación con los alumnos, formación continuada, gusto/placer/satisfacción por la docencia, relación con la investigación científica, remuneración salarial, contribución social de la actuación docente, ambiente académico, autonomía, seguridad/ estabilidad/prestigio y desafíos/compartir conocimiento. Además del diseño de una pirámide, en la cual las autoras consideraron los principales motivadores de la permanencia en la profesión docente en la percepción de los profesores entrevistados, jerarquizados por la cantidad de apariciones. Según los autores, la investigación permitió que emergieran factores motivacionales mayormente intrínsecos y relacionados a la teoría de autodeterminación, además de poder jerarquizarlos en orden de relevancia.

Como se puede apreciar, los métodos cualitativos sin tener preguntas cerradas y específicas se obtienen factores en concordancia con la teoría que surgen en el desarrollo de cada respuesta abierta de las entrevistas, haciendo que los factores encontrados sean propios de la persona y no un encajamiento entre opciones ya predeterminadas. 


\section{Consideraciones Finales}

Las personas, por la propia interacción social y las ganas de crecer personal y socialmente, tienden a escoger una profesión universitaria para estudiar. Dicha elección está asociada a múltiples factores, los cuales pueden ser personales (intrínseco) o sociales (extrínseco) y la meta principal es lograr una formación, que, por la dinámica social, será académica y personal.

La idea de formación, se entiende como un proceso complejo, formados por tres movimiento: la autoformación, la heteroformación y la ecoformación, los cuales propician la adquisición de nuevo conocimiento, reorganizando el propio saber, en medio de permanentes transformaciones del conocimiento, generando una formación, inmersa en un proceso permanente adaptado a la realidad que se vive.

La formación universitaria, necesita de una motivación hacia el estudio, la cual puede ser por factores externos o por una motivación interna, la cual genere un comportamiento interpersonal positivo, que promueve la autonomía, propiciando un desarrollo completo en las tareas, persistencia, concentración, desconexión con el tiempo invertido en las actividades y placer. Por otra parte, la motivación por medios externos proviene de la familia, la sociedad, la escuela y de forma preestablecida, también depende de las políticas públicas que cada nación adopta para ofrecer estudios universitarios a sus habitantes.

Cuadro 1. Cuadro comparativo entre la metodología cualitativa y cuantitativa utilizada en estudios sobre motivación.

\begin{tabular}{|c|c|c|}
\hline & \multicolumn{2}{|c|}{ Metodología } \\
\hline & Cuantitativo & Cualitativo \\
\hline Métodos & Encuestas con escalas tipo likert & Entrevistas semi-estructuradas \\
\hline Organización & Orienta las preguntas que se realizan & $\begin{array}{l}\text { Contribuye para dar explicación y validar las } \\
\text { categorías que surgen o emergen del análisis }\end{array}$ \\
\hline Tratamiento de los resultados & De tipo estadístico & $\begin{array}{l}\text { Análisis de Contenido, en la mayoría de los } \\
\text { casos }\end{array}$ \\
\hline Resultados & $\begin{array}{l}\text { En función de los resultados estadístico } \\
\text { y comparaciones }\end{array}$ & $\begin{array}{l}\text { Comprensiones a partir de las categorías que } \\
\text { emergen y la fundamentación teórica que los } \\
\text { sustenta. }\end{array}$ \\
\hline
\end{tabular}

Las investigaciones pueden ser de orden cualitativo o cuantitativo en muchas áreas del conocimiento, los estudios sobre motivación no escapan de esa realidad, en este trabajo se 
pudo observar las diferencias que se disponen en ambos tipos de metodología y pueden ser observadas en el cuadro comparativo 1. Las diferencias no son solo en el método de recolección de datos, sino en el análisis y tratamiento de la información.

Los estudios realizados sobre la motivación asociada a cursar estudios universitarios, en su mayoría, son de tipo cuantitativo, con uso de escalas tipo likert, enunciados y análisis enmarcados en bases teóricas especificas. Entre las fundamentaciones teóricas utilizadas esta la dicotomía entre la motivación intrínseca y extrínseca, el modelo continuum de autodeterminación, la teoría de expectación/valor y el modelo de metas.

Los estudios cuantitativos permiten obtener una visión generalizada de la motivación que presentan los estudiantes para estudiar una carrera profesional, perdiendo detalles que permitan hondar sobre como los factores sociales que inciden en la persona y su autonomía de acción.

Por otra parte, los estudios cualitativos en motivación, a pesar de ser muy pocos, permiten tener una visión de las personas participantes, como sujetos complejos y dinámicos, además de conocer a partir de sus experiencias que lo constituyen individual y socialmente, cada factor motivacional que está presente, sin requerir que el sujeto se deba encajar entre una lista de opciones que le son presentadas en una encuesta cerrada. Teniendo resultados que se correlacionan con los sujetos que participaron en la investigación y que no están apartados de las teorías pre-existentes sobre motivación.

\section{Referências}

BARDIN Laurence. Análise de conteúdo. Edições 70. 1977

BERNAL-BECERRIL M.L, GODÍNEZ-RODRÍGUEZ, M.A. Aprendizaje del cuidado obstétrico, experiencia de los estudiantes de enfermería en la práctica clínica. Enfermería Universitaria. v.13, n. 4, p.:233---238 oct. 2016. Disponible en: <http://www.revistas.unam.mx/index.php/reu/article/view/57859/51262> accesado: 01 oct 2018

BRAVO, G., VERGARA, M. Factores que determinan la elección de carrera profesional: en estudiantes de undécimo grado de colegios públicos y privados de Barrancabermeja. Revista Psicoespacios, v. 12, n. 20, pp.35-48, feb. 2018.. Disponible en https://doi.org/10.25057/issn.2145-2776 
BZUNECK, José Aloyseo; GUIMARAES, Sueli Édi Rufini. Estilos de professores na promoção da motivação intrínseca: reformulação e validação de instrumento. Psic.: Teor. $e$ Pesq., Brasília , v. 23, n. 4, p. 415-421, dic. 2007 . Disponible en $<\mathrm{http}: / /$ www.scielo.br/scielo.php?script=sci_arttext\&pid=S0102$37722007000400007 \& \operatorname{lng}=$ es\&nrm=iso $>$ Acceso en 04 mar. 2018

CANALES, Manuel. Metodologias de investigación social. Santiago: Lom Ediciones, 2006. Construcción de cuestionarios y escalas: El proceso de La producción de información cuantitativa.

CANO CELESTINO, María Alicia. Motivación y elección de carrera. Rev. Mex. Orient. Educ., México , v. 5, n. 13, p. 6-9, fev. 2008 . Disponível em $<$ http://pepsic.bvsalud.org/scielo.php?script=sci_arttext\&pid=S1665$75272008000100003 \& \operatorname{lng}=$ pt\&nrm=iso>. acessos em 29 jul. 2018.

CHIROLEU, Adriana. La inclusión en la educación superior como política pública: tres experiencias en América Latina. Revista Iberoamericana de Educación, v. 48, n. 5, Madrid, OEI, 2009. Disponible en: < https://rieoei.org/historico/deloslectores/2740Chiroleu.pdf >. accedido en 06 sept. 2018

DAVOGLIO, Tárcia Rita; SPAGNOLO, Carla; SANTOS, Bettina Steren dos. Motivação para a permanência na profissão: a percepção dos docentes universitários. Psicol. Esc. Educ., Maringá, v. 21, n. 2, p. 175-182, agosto 2017 . Disponible en $<$ http://www.scielo.br/scielo.php?script=sci_arttext\&pid=S1413$85572017000200175 \& \operatorname{lng}=$ es\&nrm=iso>. accedido en 01 oct. 2018

Diario Oficial da União. Seção 3. № 55, quarta-feira, 21 de março de 2018. P.53. disponible en <http://download.inep.gov.br/educacao_basica/enem/edital/2018/edital_enem_2018.pdf> accesado el: 15 de oct. 2018, 11:17

DOS SANTOS, Bettina Steren, RODENBUSCH Camila de Barros y ANTUNES Denise Dalpiaz. Aspectos motivacionais da profissão docente Universitária. Revista Educação Temática Digital, Campinas, v.10, n.esp., p.294-312, out. 2009. Disponible en 
<http://scholar.google.com.br/scholar_url?url=https://dialne

t.unirioja.es/descarga/articulo/4856467.pdf\&hl=en\&sa=X\&scisig=AAGBfm2iG3B13oq46im

J2Yfkc_MKt-Q8jA\&nossl=1\&oi=scholarr>, accesado: 01 oct 2018

ECCLES, Jacquelynne; WIGFIELD, Allan. "In the Mind of the Actor: The Structure of Adolescents' Achievement Task Values and Expectancy-Related Beliefs." Personality and Social Psychology Bulletin. N. 21, v. 3, p. 215-225. 1995. Disponible en: http://hdl.handle.net/2027.42/69045. accesado 04 ago. 2018

ECHEVERRI-SÁNCHEZ, Laura. VALENCIA-ARIAS, Alejandro. BENJUMEA-ARIAS, Martha y BARRERA-DEL TORO. Andry. Factores que inciden en la intención emprendedora del estudiantado universitario: Un análisis cualitativo. Revista Electrónica Educare, v. 22, n. 2, p.1-19, ago. 2018. Disponible en < https://dialnet.unirioja.es/descarga/articulo/6331058.pdf> accesado: 01 oct 2018

ELVIRA-VALDÉS, M. y PUJOL, L. Variables cognitivas e ingreso universitario: predictores del rendimiento académico. Universitas Psychologica, 13(4), 1557-1567 (2014) Disponible en <http://www.scielo.org.co/pdf/rups/v13n4/v13n4a26.pdf>. accedido en 06 sept. 2018

FIGUEROA E. La elección de carrera: una decisión de gran trascendencia. Educación. . v. 2, n. 3, mar 1993, 29 jul 2018 Disponible en: https://dialnet.unirioja.es/descarga/articulo/5056955.pdf, accesado: 01 oct 2018

GARCES, Carlos René Rodríguez; FUENTES, Geraldo Padilla. Elección profesional y sesgo de selección: evaluación de los sistemas de admisión universitaria en Chile en un contexto de agenda pro-inclusión. Avaliação (Campinas), Sorocaba, v. 22, n. 3, p. 852-870, Dec. 2017 - disponible en <http://www.scielo.br/scielo.php?script=sci_arttext\&pid=S141440772017000300852\&lng=en\&nrm=iso>. accesado 06 Sept. 2018

GARCIA GARDUNO, José María; ORGANISTA SANDOVAL, Javier. Motivación y expectativas para ingresar a la carrera de profesor de educación primaria: Un estudio de tres generaciones de estudiantes normalistas mexicanos de primer ingreso. REDIE, Ensenada, v.
$8, \mathrm{n}$.
2, p.
$1-17$,
2006
Disponible
en 
$<$ http://www.scielo.org.mx/scielo.php?script=sci_arttext\&pid=S1607-

40412006000200008\&lng=es\&nrm=iso>. Acceso en 04 mar. 2018

GRINER, Almog; SAMPAIO, Luciano Menezes Bezerra; SAMPAIO, Raquel Menezes Bezerra. A política afirmativa "Argumento de Inclusão" como forma de acesso à universidade pública: o caso da Universidade Federal do Rio Grande do Norte. Rev. Adm. Pública, Rio de Janeiro, v. 49, n. 5, p. 1291-1317, Oct. 2015. disponible en $<$ http://www.scielo.br/scielo.php?script=sci_arttext\&pid=S0034-

76122015000501291\&lng=en\&nrm=iso>. accedido 06 Sept. 2018.

JOLY, Maria Cristina Rodrigues Azevedo; PRATES, Eli Andrade Rocha. Avaliação da Escala de Motivação Acadêmica em estudantes paulistas: propriedades psicométricas. PsicoUSF (Impr.), Itatiba , v. 16, n. 2, p. 175-184, Aug. 2011 . Available from $<$ http://www.scielo.br/scielo.php?script=sci_arttext\&pid=S141382712011000200006\&lng=en\&nrm=iso>. accessado 03 Aug. 2018

KILINÇ, Hamed WATT, Helen y RICHARDSON, Paul. Factors Influencing Teaching Choice inTurkey. Asia-Pacific Journal of Teacher Education, v. 40, n. 3, p. 199-226, jul 2012. Disponible en: http://dx.doi.org/10.1080/1359866X.2012.700048. accedido 06 agost. 2018

GUIMARÃES, S., BZUNECK, J. \& JOLY, M. (2010). Escala de Avaliação da Motivação para Universidade (AMU).Manuscrito não publicado. Universidade Estadual de Londrina / Universidade São Francisco.

MONTALVO-AGUILAR, Michell et al . Desempeño académico en el primer año de medicina: relación con intereses vocacionales, actitud ante el estudio y fuerza de motivación. FEM (Ed. impresa), Barcelona , v. 16, n. 2, p. 111-117, jun. 2013. Disponible en $\quad<$ http://scielo.isciii.es/scielo.php?script=sci_arttext\&pid=S2014$98322013000200010 \& \operatorname{lng}=$ es\&nrm=iso>. Acceso en 10 abri. 2018

PEREZ DE PEREZ, Anneris; DIAZ, María Elena. La prueba de aptitud académica: una visión de la subprueba de comprensión de lectura. Investigación y Postgrado, Caracas, v. 21, n. 2, p. 143-176, oct. $2006 \quad$. Disponible en 
<http://www.scielo.org.ve/scielo.php?script=sci_arttext\&pid=S1316-

00872006000200006\&lng=es\&nrm=iso>. accedido en 06 sept. 2018

PINEAU, Gaton. A formação em dois tempos, três movimentos. IN: Temporalidades na formação: rumo a novos sincronizadores. Traduçõa L. P. de Souza. São Paulo: Triom. 2003. p. $151-164$

PINTRICH Paul. A Motivational Science Perspective on the Role of Student Motivation in Learning and Teaching Contexts. Journal of Educational Psychology. v. 95, n. 4, p. 667-786, jun. 2003. Disponible

en <https://ueeval.ucr.edu/teaching_practices_inventory/Pintrich_2003.pdf> Acceso en 15 jun. 2018

REEVE Jonhmarshall, DECI Edward, RYAN Richard. Self-determination theory: A dialectical framework for understanding sociocultural influences on student motivation. 2004. Disponible en <https://www.researchgate.net/publication/309563565>. Acceso en: 20 junio. 2018.

MORIN, Edgar. Los sietes saberes necesarios a la educación del futuro. UNESCO.1999

RYAN, Richard y DECI, Edward. La Teoría de la Autodeterminación y la Facilitación de la Motivación Intrínseca, el Desarrollo Social, y el Bienestar. American Psychologist. V. 5, n. 1, p. 1-16, jan. 2000. Versión traducida al español. Disponible en <https://selfdeterminationtheory.org/SDT/documents/2000_RyanDeci_SpanishAm Psych.pdf > consultado: 08 oct. 2018

SANTOS, Acácia Aparecida Angeli dos; ALCARA, Adriana Rosecler; ZENORINI, Rita da Penha Campos. Estudos psicométricos da escala de motivação para a aprendizagem de universitários. Fractal, Rev. Psicol., Rio de Janeiro, v. 25, n. 3, p. 531-546, Dec. 2013 . Available from $\quad<$ http://www.scielo.br/scielo.php?script=sci_arttext\&pid=S198402922013000300008\&lng=en\&nrm=iso>. access on 03 Aug. 2018. 
SARRAMONA, Jaume. "La autoformación en una sociedad cognitiva". Revista Iberoamericana de Educación a Distancia (RIED), n. 2, v. 1, p 28-37, jun 1999. Disponible en: https://core.ac.uk/download/pdf/78533421.pdf> accesado 01 sep. 2018 SiSU. Disponible en < http://sisu.mec.gov.br/como-funciona> accesado el: 15 de oct. 2018, $11: 15$

STEINMANN, Andrea; BOSCH, Beatriz; AIASSA, Delia. Motivación y expectativas de los estudantes por aprender ciencias en la universidad: un estudio exploratorio. Revista Mexicana de Investigación Educativa. México v.18, n. 57, p. 585-598, 2013. Disponible en <http://www.redalyc.org/pdf/140/14025774012.pdf> Acceso en 02 mar. 2018

SURYANI, Anne \& WATT, Helen \& RICHARDSON, Paul. (2016). Students' motivations to become teachers: FIT-Choice findings from Indonesia. International Journal of Quantitative Research in Education. V. 3. N. 179. 10.1504/IJQRE.2016.077802. : Disponible en: <https://www.researchgate.net/publication/305382103> accesado 04 ago. 2018

TEIXEIRA Elizabeth. As três metodologias: academia, da ciência e da pesquisa. 11.ed.Petrópolis, RJ: Vozes, 2014

WATT, H.M.G., \& RICHARDSON, P.W. (2007). Motivational factors influencing teaching as a career choice: Development and validation of the FIT-Choice scale. Journal of Experimental Education, V. 75, p. 167-202. Disponible en <https://doi.org/10.3200/JEXE.75.3.167-202>. accesado 04 ago. 2018 